\title{
Miranda
}

Revue pluridisciplinaire du monde anglophone /

Multidisciplinary peer-reviewed journal on the English-

speaking world

1 | 2010

Variations on Darwin

\section{Darwin et la question de la finalité}

\section{Philippe Solal}

\section{(2) OpenEdition}

\section{Journals}

Édition électronique

URL : http://journals.openedition.org/miranda/330

DOI : 10.4000/miranda.330

ISSN : 2108-6559

\section{Éditeur}

Université Toulouse - Jean Jaurès

\section{Référence électronique}

Philippe Solal, « Darwin et la question de la finalité », Miranda [En ligne], 1 | 2010, mis en ligne le 23 mars 2010, consulté le 16 février 2021. URL : http://journals.openedition.org/miranda/330 ; DOI : https://doi.org/10.4000/miranda.330

Ce document a été généré automatiquement le 16 février 2021.

\section{c) (i)}

Miranda is licensed under a Creative Commons Attribution-NonCommercial-NoDerivatives 4.0 International License. 


\title{
Darwin et la question de la finalité
}

\author{
Philippe Solal
}

1 La théorie de l'évolution des espèces, que Charles Darwin élabora à partir des enseignements tirés de son voyage à bord du Beagle, intéresse au plus haut point la philosophie. La nouvelle conception de la nature, et de la place que l'homme occupe en son sein, telles qu'on peut les déduire de cette théorie, remet en effet en question à peu près tout ce que la philosophie a écrit sur ce sujet, à de rares exceptions près.

2 On comprend dès lors la nature de cet intérêt philosophique pour le Darwinisme : il est de l'ordre d'un véritable traumatisme intellectuel. Ce n'est pas seulement le message de la Bible qui est ici subverti. C'est le travail de la raison, celui que la philosophie a prétendu exercer depuis l'Antiquité, en se tenant à distance des dogmes religieux et au plus près de la rigueur de ses raisonnements.

La théorie darwinienne de l'évolution a remis en question le concept-clef qui avait permis aux doctrines philosophiques les plus reçues de penser la nature, à travers ses manifestations visibles: le concept de finalité. Avec une belle constance, qui tranche avec l'idée reçue selon laquelle les philosophes n'ont cessé de se contredire les uns les autres au cours de leur histoire, la philosophie a affirmé que la nature ne pouvait pas se penser, s'étudier, s'interpréter, sans l'aide de l'idée de finalité. L'usage de ce concept s'est en effet déployé de deux manières :

- Ou bien l'on affirme que la nature poursuit des buts que l'examen attentif des êtres vivants nous révèle aisément.

- Ou bien l'on pose que la nature est elle-même l'instrument d'un projet, le résultat d'une intention, l'expression d'un "dessein intelligent ", dont la source peut-être qualifiée de surnaturelle, source d'origine divine.

Dans le premier cas, la finalité est dite « immanente », ce qui signifie que c'est la nature considérée dans son unité, qui porte en elle et réalise des fins que la raison humaine doit découvrir. Dans le deuxième cas, la finalité se rattache à un ordre supérieur, un ordre dit «transcendant », celui du vouloir divin, et la nature est tout entière finalisée par ce Vouloir même. 


\section{Un exemple de finalisme immanent : la doctrine d'Aristote}

5 Evoquons brièvement quelques-unes de ces doctrines philosophiques. L'une des plus célèbres est certainement celle d'Aristote qui, au III siècle av. JC, donne une définition de la nature qui rompt avec les anciennes représentations du mythe grec. La nature, physis, est d'abord selon Aristote "principe de mouvement ", c'est-à-dire cette énergie à l'œuvre dans le déploiement des êtres, leur germination, leur épanouissement, et leur corruption finale. Mais ce déploiement ne peut se comprendre qu'en fonction de la direction qu'il prend. Or dans la nature chaque réalité tend à réaliser sa nature propre et sa destination finale, lesquelles ne se révèlent que lorsqu'un être parvient à atteindre son parfait état d'accomplissement.

6 Aristote répète, en particulier dans son ouvrage La Politique, que la « nature ne fait rien en vain» (Aristote 1962, 17). Elle n'accorde pas d'organes aux êtres vivants qu'ils ne pourraient pleinement exploiter pour accomplir leur nature propre, sans quoi cela serait du gaspillage. Or dans sa sagesse, la nature est économe, et elle vise juste : ainsi, si elle a attribué aux hommes des mains, organe si précieux et si spécial, c'est parce qu'il est seul capable d'en tirer le meilleur profit. En effet l'homme seul possède le logos, c'est-àdire la pensée rationnelle déployée par la parole.

7 Sa raison lui permettra donc de tirer le meilleur parti de cet organe de préhension, qui lui donne la capacité de fabriquer des outils. Avec ces outils l'homme bâtira des Cités (Polis) dans lesquelles on construira, entre autres choses, des Parlements, autrement dit des lieux où l'on parlera de ce qu'il est bien de faire pour la communauté tout entière. Alors l'homme réalisera sa fin, ce à quoi il est par nature destiné. Il réalisera la forme la plus accomplie de son essence, en parvenant jusqu'à la dimension de citoyen, car, selon Aristote «l'homme est par nature un animal politique » (Aristote 1962, 53) ${ }^{1}$.

On le voit, la conception aristotélicienne de la nature se dédouble en deux concepts qu'il s'agit de penser solidairement : la nature est à la fois la force et le principe de la croissance des êtres (ce que confirme l'étymologie du mot physis, qui dérive du verbe phuein : croître, se développer); mais elle est aussi l'essence spéciale de chaque réalité. Dans les deux cas, c'est le concept de finalité qui permet de penser la nature, car en définitive, la vraie nature d'un être se confond avec son but. Mais il faut observer que cette conception de la nature ne fait pas intervenir la volonté d'une divinité, puisque Dieu, chez Aristote, ne s'occupe pas des hommes et n'intervient jamais dans le cours des événements naturels. Aussi Aristote nous expose-t-il une nature dont la finalité est immanente. En effet, le Dieu Aristotélicien n'est pas créateur. Il a juste donné l'impulsion première au mouvement de la nature, et c'est pourquoi Aristote le nomme le « Premier Moteur » (Aristote 1999, 62) ${ }^{2}$.

9 Cette représentation duale de la nature (la nature comme force de mouvement et comme essence spécifique), est propre à l'élaboration philosophique de ce concept. On ne la retrouve pas dans la manière dont la science va à son tour conceptualiser la nature à partir de la Renaissance. On la reconnaît par contre chez des philosophes bien postérieurs à Aristote : ainsi, au XVII ${ }^{\mathrm{e}}$ siècle, Spinoza distingue dans l'Ethique la « nature naturante " (natura naturans) de la "nature naturée» (natura naturata), c'est à-dire entre la nature comme force de production et la nature comme essence (Spinoza 1677, 8). 


\section{Un exemple de finalisme transcendant : la philosophie chrétienne}

10 Les philosophies qui fleurissent au Moyen-Âge se distinguent des précédentes en ce qu'elles placent au contraire la finalité dans une transcendance, celle du Dieu judéochrétien, fidèles en cela au message des Saintes Ecritures. Ces doctrines qui composent ce que les historiens des idées ont appelé "a philosophie chrétienne », celle de SaintAugustin ou de Thomas d'Aquin par exemple, tentent de retrouver par la raison ce que la foi nous révèle : la nature est le plan de Dieu, et l'homme en est le couronnement. Elles doivent répondre en particulier à cette impérieuse question : pourquoi Dieu a-t-il créé la nature et l'homme? Dans sa formulation la plus radicale, celle qu'adopta au $\mathrm{XVII}^{\mathrm{e}}$ siècle le philosophe allemand Leibniz, cette question s'exprime ainsi : « Pourquoi y a-t-il quelque chose plutôt que rien ?»(Leibniz 1714, 21) ${ }^{3}$.

11 La réponse que Leibniz donne à cette interrogation est conforme au dogme biblique : Dieu aurait pu ne rien créer, et cela n'aurait rien enlevé à sa perfection, lui dont la puissance est infinie. S'il a créé la nature, et l'homme à son sommet, c'est par amour, par pur amour. Cet amour se manifeste par la volonté qui est celle de Dieu de faire sortir l'homme du Néant pour l'inscrire dans l'Etre. Car être est une perfection plus grande que ne pas être. Cette finalité-là est transcendante.

Beaucoup d'autres doctrines pourraient être convoquées pour illustrer le finalisme transcendant. Même les philosophes des Lumières, eux pourtant si critiques à l'égard des religions, ont adopté cette position. Rousseau, Voltaire et Diderot, ont tenté de substituer à la théologie révélée par les Ecritures, la «théologie naturelle » ${ }^{4}$ qui consiste à déduire de l'observation de la nature, de l'examen de son ordre et de sa beauté, la marque de Dieu. Tout, du grain de sable à l'étoile, témoigne de Sa Présence et de Sa Sagesse.

Le philosophe allemand Kant développera même une « téléologie de la nature ", c'est-àdire qu'il s'efforcera de légitimer l'étude rationnelle de la fin (télos) que la nature poursuit, et que l'histoire nous révèle. La nature, soutient Kant dans le Projet de paix perpétuel, œuvre par divers moyens au mélange et métissage des peuples, s'efforçant de les sortir de leur isolement originaire (Kant 2007, 192). Par ce biais, elle favorise le cosmopolitisme, c'est-à-dire l'instauration future d'un État mondial unique qui mettra fin aux guerres entre nations. Par une inégale répartition géographique de ses ressources, mais aussi par les courants marins qui permettent une communication entre les peuples autochtones forcés de commercer entre eux, la nature favorise l'instauration d'un Etat unique seul promoteur du droit intégral. En effet, quand il n'y aura plus de nations pour se faire la guerre, l'homme pourra jouir de la paix perpétuelle et régler les conflits entre individus par simple police.

\section{La nature selon Darwin}

14 On comprend mieux, à travers l'évocation de ces quelques traditions de pensée, la nature du bouleversement que produisirent les thèses développées par Charles Darwin à la publication de L'Origine des espèces en 1859. Les observations du naturaliste anglais lors de son voyage sur le Beagle, les idées qu'il tire des techniques de sélection 
artificielle utilisées par les éleveurs, l'influence des thèses de Malthus sur sa propre recherche, ont amené ce dernier, on le sait, à dresser un tableau de la nature qui s'écarte radicalement du finalisme philosophique et religieux. Le Darwinisme nous décrit en effet un pur mécanisme.

Qu'est-ce que la nature ? Dans L'Origine des espèces, Darwin répond par ces mots : « But I mean by Nature, only the aggregate action and product of many natural laws, and by laws the sequence of events as ascertained by us » (Darwin 1872, 93). Certes, Descartes ne disait pas autre chose deux siècles plus tôt, quand il rappelait à ses lecteurs, dans le Discours de la Méthode, que la nature n'était pas une déesse, mais qu'elle devait être conçue comme la totalité de la matière et l'ensemble des lois qui en régissait les mouvements (Descartes 2000). Toutefois Descartes finissait par rattacher cette «mécanique » qu'est la nature à un ordre finalisé qui lui était supérieur et antérieur, celui de Dieu. "Dieu nous a fait comme nous faisons les machines", écrivait-il dans le Traité de l'Homme (Descartes 1953, 124). On se trouvait alors confronté à deux plans : celui du fonctionnement aveugle de la nature, aveugle car pure mécanicité, pure déclinaison de causes et d'effets ; et celui de la finalité et du vouloir divin.

Comment ces deux plans étaient-ils pensés ensemble par les philosophes classiques ? En concevant la nature entière comme une machine, dont le fonctionnement autonome était indépendant de toute intervention extérieure, des penseurs comme Descartes, Leibniz ou Voltaire, ont fait de Dieu un horloger. On se souvient de la phrase de Voltaire: «L'univers m'embarrasse, et je ne puis songer que cette horloge existe et n'ait point d'horloger" (Voltaire 225). Or il arrive parfois que le mécanisme de l'horloge se détraque, dysfonctionne. Ces erreurs de mécanique, la Renaissance les a dépistées dans la «monstruosité », c'est-à-dire dans l'enfantement d'individus affectés par ce que l'on appelle aujourd'hui des aberrations génétiques : nains, enfants-loups ou siamois, sujets atteints à la naissance d'une extrême difformité du corps, etc. La « leçon des monstres " nous place alors devant une nature que Dieu a vraiment désertée, comme si l'ingénieur en chef avait délaissé la surveillance de la machinerie et de ses matrices de production. Mais loin de fournir des arguments à l'athéisme, l'existence des monstres a bien plutôt renforcé l'opposition de la nature et de la grâce, chez les principaux philosophes théistes de l'âge classique.

En effet, pour compenser les erreurs de fonctionnement de cette machinerie qu'on nomme la nature, Dieu se réserve le droit de procéder à des "interventions spéciales " - les miracles. Autrement dit, la monstruosité est l'anti-miracle, le miracle inversé, le signe de la désertion de Dieu, là où le miracle pose sa toute-puissance et sa touteprésence. Une fois encore, dans cette option de pensée, le mécanisme (celui de la nature) est rattaché au finalisme (celui de l'action de Dieu), dans lequel il finit par se dissoudre. Et l'existence des miracles se présente alors comme le triomphe du vouloir divin sur le mécanisme aveugle de la nature.

\section{La rupture épistémologique}

C'est avec cette manière de penser, c'est-à-dire cette subordination du mécanisme naturel au finalisme divin, que Darwin va rompre de manière radicale. Et en ce sens, Darwin restera jusqu'au bout un scientifique exclusif, là où le Descartes physicien a laissé le pas au Descartes métaphysicien. Darwin nous apprend en effet que la nature biologique produit de la diversité et de la variabilité : variétés au sein d'une même espèce et 
diversité d'espèces. Il nous enseigne que seules demeurent, au sein de cette variabilité, les espèces les plus aptes à survivre à l'environnement et à la lutte pour la vie («struggle for life») contre d'autres espèces. Les autres sont impitoyablement éliminées, et Darwin nomme ce processus d'élimination «sélection naturelle ». Or ce processus de sélection est un pur mécanisme, ce qui signifie que ne s'y repèrent aucune finalité, aucune intention, aucune planification, mais bien plutôt de la contingence et du hasard.

Que la chaleur augmente sur l'une des îles des Galápagos, et que sous l'effet de cette modification du climat les coques des graines dont se nourrissent les pinsons deviennent plus résistantes, il y a là rupture d'un équilibre antérieur et seuls les pinsons au bec plus large et plus épais, assez solide en tout cas pour mieux casser les coques, résisteront à cette modification du milieu. Ils seront même avantagés par leur complexion initiale, tandis que les autres variétés de pinson seront éliminées. Ce qui vaut pour une variété vaudra pour les espèces, car comme le répète Darwin, les variétés sont des espèces naissantes.

Or ce processus est contingent. Il aurait pu ne pas se produire, et d'autres modifications du milieu auraient pu permettre le triomphe d'autres variétés, ouvrant de proche en proche, ou de loin en loin, la voie à l'émergence d'autres espèces. Voilà ce qu'est le mécanisme darwinien: la description d'une évolution où rien n'est planifié. Nulle direction ne vient préalablement dessiner un progrès. Le schéma de l'arbre darwinien indique des embranchements qui sont autant de cul-de-sac et d'impasses. Et l'on sait que les intuitions de Darwin ont été confirmées par la génétique du $\mathrm{XX}^{\mathrm{e}}$ siècle, qui a su mettre en lumière certaines causes initiales de la variabilité du vivant. La variabilité provient de processus aléatoires, d'erreurs de frappe dans la duplication de l'ADN au moment de la reproduction.

Ces erreurs sont fécondes, car elles produisent des modifications morphologiques sur lesquelles va s'opérer ensuite l'action de la sélection naturelle. Ainsi, à la contingence des ruptures d'équilibres intervenant dans les processus sélectifs, la biologie moderne a rajouté l'aléatoire des processus de duplication génétique. Dès lors, l'ordre naturel n'est plus un donné. L'organisation du vivant est pétrie de désordres, de déviances créatrices, d'irrégularités fécondes. La capacité d'auto-organisation des êtres vivants résulte bien plutôt de leur capacité à faire face à des agressions aléatoires et l'émergence des formes naturelles se nourrit de l'imprévu et du chaos.

\section{Darwin et la question de la finalité}

Darwin est donc celui qui a débarrassé l'idée de nature de celle de finalité. Lui qui fut séduit lors de ses années de formation par la théologie naturelle d'un William Paley, empreinte de finalisme, s'est tournée vers la positivité d'un pur mécanisme. La métaphysique le répugne, et il refuse de s'aventurer dans les questions si obscures et insondables qu'elle soulève, celles des origines et du sens de tout ce processus qu'on appelle la nature. Même Newton n'était pas allé aussi loin ${ }^{5}$.

Pourtant, dans l'exposition même de sa théorie, il a dû constamment se battre contre la prééminence de ce concept dans notre manière la plus usuelle de parler de la nature. Tout se passe comme si la question de la finalité avait fini par resurgir, venant polluer le discours même qui avait tenté d'en éliminer la pertinence. Ainsi, dans un important 
passage de l'édition de 1872 de L'Origine des espèces, au chapitre IV, Darwin souligne que plusieurs écrivains ont mal compris ce terme de " sélection naturelle » :

Several writers have misapprehended or objected to the term Natural Selection. Some have even imagined that natural selection induces variability, whereas it implies only the preservation of such variations as occur and are beneficial to the being under its conditions of life. No one objects to agriculturists speaking of the potent effects of man's selection; and in this case the individual differences given by nature, which man for some object selects, must of necessity first occur. Others have objected that the term selection implies conscious choice in the animals which become modified; and it has even been urged that, as plants have no volition, natural selection is not applicable to them! In the literal sense of the word, no doubt, natural selection is a false term ; but who ever objected to chemists speaking of the elective affinities of the various elements?-and yet an acid cannot strictly be said to elect the base with which it in preference combines. It has been said that I speak of natural selection as an active power or Deity ; but who objects to an author speaking of the attraction of gravity as ruling the movements of the planets? Everyone knows what is meant and is implied by such metaphorical expressions; and they are almost necessary for brevity. So again it is difficult to avoid personifying the word Nature ; but I mean by Nature, only the aggregate action and product of many natural laws, and by laws the sequence of events as ascertained by us. With a little familiarity such superficial objections will be forgotten. (Darwin 1872, 93).

La « sélection naturelle » implique, en effet, l'idée d'un choix conscient et volontaire, donc d'un processus finalisé. C'est pourquoi Darwin précise que dans le sens littéral du mot, le terme de sélection naturelle est sans doute erroné, et l'expression métaphorique n'est utilisée que pour la clarté de la discussion.

Ces difficultés de formulation, qui consistent à décrire un mécanisme en utilisant des expressions renvoyant à des actions finalisées, se retrouvent dans bien d'autres occurrences de l'œuvre de Darwin. Quand celui-ci parle de la sélection naturelle comme "d'une force » ou d'une "puissance naturelle ", quand il évoque les espèces «que la sélection naturelle a produite ", il est dans la métaphore, celle qui consiste à faire de la nature un sujet. Michel Prum ${ }^{6}$, l'un des traducteurs contemporains de Darwin en France, a insisté sur ce point : notre discours usuel nous a habitués à considérer la nature comme un "agent» et il est très difficile d'échapper à ces tournures intellectuelles. Darwin lui-même n'a pu faire autrement que d'exprimer sa théorie en « langage pré-darwinien ", même si par la suite il a remplacé l'expression « sélection naturelle » par celle, moins ambiguë, de « survie du plus apte».

Il est vrai que l'expression «sélection naturelle» est d'abord une métaphore, qui emprunte son modèle de comparaison à la sélection artificielle des éleveurs de pigeons, consciente, volontaire et finalisée celle-là. Mais ce problème de terminologie n'est pas spécifique à Darwin. Au XX ${ }^{\mathrm{e}}$ siècle, les généticiens se heurteront à la même difficulté. La notion de "programme génétique » fait conceptuellement référence à la finalité, celle d'une programmation. Quand, au début des années 1970, le généticien français François Jacob développe sa thèse selon laquelle le vivant fait du « bricolage ", en réutilisant des éléments du génome autrefois affectés à des fonctions organiques différentes, c'est-àdire en faisant du "neuf avec du vieux», il se heurte au même problème (Jacob 55). François Jacob a alors conscience d'utiliser un vocabulaire en réalité inadéquat: affirmer que la "nature bricole » c'est en même temps livrer une description erronée car quand on bricole, déclare-t-il, le bricoleur sait ce qu'il a dans la tête, alors que l'évolution procède en aveugle. 


\section{Finalité interne et finalité externe}

Pourquoi, en biologie, ne parvient-on pas à mettre en forme un discours intégralement mécaniste sur le vivant, comme la science a su le faire pour la physique? Cette difficulté ne profite-t-elle pas aux créationnistes qui tentent de s'opposer à la vision purement mécaniste de la nature que la science déploie sous nos yeux?

Plusieurs réponses pourraient être suggérées ici pour mesurer le sens et la portée de cette difficulté. Dans le cas des éléments chimiques dont parle la physique nous n'avons pas de mal à discerner la métaphore, quand par exemple nous parlons de "l'affinité " des acides et des bases, comme le rappelle Darwin lui-même (Darwin 1859, 93). Dans le cas des êtres vivants, la métaphore est beaucoup moins perceptible et le risque de se laisser piéger par les mots est bien plus grand. La raison provient du fait que la nature auquel le biologiste s'intéresse porte en particulier sur des êtres organisés, c'est-à-dire constitués d'organes. Or, ce qui définit un organe c'est la fonction qu'il assume au sein d'un organisme, et on ne peut parler de fonction sans réintroduire le concept de finalité. La fonction du rein est de purifier le sang. Le «but de fonctionnement du rein » est cette purification même. Comment alors parler du vivant sans parler de finalité ?

C'est là que s'opère une distinction entre deux types de finalité, distinction déjà posée par Kant au XVIII siècle dans la Critique de la faculté de juger entre la finalité interne (celle qui permet de décrire le fonctionnement d'un organe) et la finalité externe. Les animaux possèdent des organes qui répondent à des fonctions spécifiques. Ces fonctions peuvent être considérées comme la "finalité interne " de l'organe destiné à les remplir. Par contre les êtres vivants n'obéissent à aucune finalité externe, transcendante au fonctionnement de chaque organe en particulier, comme le serait par exemple l'affirmation selon laquelle «l'homme est fait pour coloniser et régner sur la Terre " (Kant 1993, 289).

Si l'on admet la pertinence de cette distinction entre finalité interne et finalité externe, on comprend pourquoi il est si difficile d'échapper au langage de la finalité pour parler de la nature. L'organisation biologique des espèces fait appel à une finalité interne qu'il est tentant de transformer en finalité externe, en s'échappant du cadre strict $d u$ fonctionnement d'un organe. Dès lors on franchit la ligne jaune, on réintroduit de la métaphysique au cœur de la science positive, ce que le Darwinisme a précisément tenté d'interdire.

\section{L'illusion d'une temporalité tronquée}

Une autre raison peut-être avancée pour expliquer la persistance de la question de la finalité au sein de la biologie contemporaine. Même en décortiquant les différents éléments du mécanisme de la nature lors de son évolution, comme a su admirablement le faire Darwin, nous ne pouvons pas nous déprendre d'une illusion de perspective, qui est le propre de notre condition de sujet connaissant. L'espèce humaine est d'apparition récente, la civilisation a tout au plus six mille ans, et ce que nous appelons la science date de quatre-cents ans. Notre regard porte donc sur des périodes très courtes, ce qui nous donne l'illusion d'un monde figé, constitué d'essences éternelles, là où en réalité tout est en devenir instable. Cette instabilité ne se révèle pleinement qu'à 
des échelles de temps bien plus grandes, lorsque nous nous reculons. Sur cinquante millions d'années, le procès de la nature est bien plus instructif.

Ignorant des véritables processus de variation et d'élimination qui scandent le passé et fournissent la trame du devenir, nous croyons que l'ordre perçu est le produit d'une nature intelligente, providentielle, inventive, pourvoyeuse et ingénieuse, sans apercevoir l'envers du décor. Cet « envers » est fait de ratages, de formes improbables, d'essais infructueux, d'aberrations mort-nées. L'envers et l'endroit. Nous sommes comme ce joueur qui, sur le millier de billets de loterie achetés, croit en sa chance et loue la providence divine parce que l'un d'eux est gagnant, oubliant que les neuf cent quatre-vingt-dix-neuf autres étaient perdants. L'illusion d'optique dont nous sommes victimes est un peu du même ordre. Par défaut de sens de la temporalité, par une conscience bien trop étroite du temps, nous ne sommes pas en mesure de percevoir le chaos du devenir et son improbable errance. Or, cette instabilité et cette errance sont précisément ce que ni la philosophie ni la religion ne peuvent penser. Nous nous gargarisons alors de la sagesse de la nature, c'est-à-dire d'un état transitoire dans un océan de fluctuations que nous qualifions improprement d'ordre finalisé et définitif.

Cette illusion d'optique nous inscrit dans une nature qui prend des traits plus rassurants, et nous interprétons l'ébauche d'ordre que nous y percevons en terme d'absolu. Enfants du hasard, nous nous croyons fils de la nécessité, et cette croyance nous permet de chercher un sens global à l'existence. Tel serait le propre de notre condition tout entière sise sur cette illusion fondamentale que le Darwinisme a bousculé, pour le meilleur et pour le pire.

\section{Nietzsche et Darwin}

Doit-on conclure de ce qui précède que le Darwinisme, en expulsant de son horizon le concept de finalité, n'a pu donner naissance à aucune philosophie qui soit capable de le penser, et de l'intégrer dans un système de pensée digne de ce nom ? Doit-on faire du Darwinisme l'inassimilable de la philosophie, conséquence du traumatisme intellectuel que nous évoquions en ouverture? Rien n'est moins faux si l'on se rappelle que l'une des philosophies les plus fascinantes du XIX ${ }^{e}$ siècle, qui a irradié de son influence tout le $\mathrm{XX}^{\mathrm{e}}$ siècle, celle de Nietzsche, s'inspire directement des résultats de la théorie de l'évolution.

Nietzsche a pris acte très tôt du bouleversement qu'allait produire le Darwinisme sur la vision religieuse de la nature. Avec Darwin, la nature n'est plus cette œuvre parfaite dont parle la religion, mais le lieu d'une inlassable cruauté. Dans la nature, les grands mangent les petits, le loup dévore l'agneau, les forts écrasent les faibles, et ne subsistent dans l'être que ceux qui ont assez de vitalité pour survivre à un impitoyable processus de sélection naturelle. Est-ce là l'œuvre parfaite d'un Dieu amour ? Sûrement pas. Désormais, les êtres vivants n'apparaissent plus d'un coup comme dans le récit de la Genèse, mais sont le fruit d'une lente évolution. Avec Darwin, en effet, il n'y a plus de « Dieu magicien » qui, par la seule force d'une Parole créatrice fait apparaître les êtres, à la manière d'un magicien dont les formules accompagnent les tours.

La nature apparaît enfin sous son vrai jour, comme le lieu d'une lutte incessante pour la survie, où tout est hostilité, menace, souffrance. L'épine et le chardon ne sont pas les conséquences d'un châtiment divin, comme nous le fait croire la Bible, à la suite de 
quelque imaginaire péché originel. Rien n'est fait au départ pour l'homme, et s'il peut trouver de quoi favoriser sa subsistance, la nature regorge aussi de pièges, et de dangers, de plantes vénéneuses et de poisons. C'est au prix de mille sacrifices qu'il la dominera, écartant de sa route les substances et les espèces qui peuvent nuire à sa propre conservation.

La philosophie de Nietzsche a su récupérer les intuitions fondamentales du Darwinisme pour dresser un nouveau tableau du «sens de l'Être». Nietzsche pose comme fondement et essence du réel, comme unique réalité fondamentale, une Force à la fois créatrice et destructrice qu'il appelle "Volonté de puissance ». Les mots ici ne doivent pas nous tromper : cette expression ne désigne pas le projet (la "volonté ») d'un Dieu, mais une dynamique qui est le Réel, « une énergie qui se meut sans but, sans projet, sans intention " (Nietzsche 1886, 36). N'est-ce pas cette Force d'expansion que nous met sous les yeux Darwin, à travers le jeu de cette pièce unique qu'est la lutte pour l'existence de tous contre tous?

Cette Force, la Volonté de puissance, est poussée de domination et, en tant que telle, elle est à elle-même sa propre fin. Poussée qui anime la conduite de toutes les formes vivantes, aussi bien les plantes, les espèces animales que l'homme lui-même. Car tous les êtres vivants possèdent cette Force qui se démultiplie ainsi à travers eux, cette poussée que l'on nomme pulsion. Dans le monde du vivant la prégnance de cette Force mesure la vitalité, laquelle se combine avec une fureur destructrice qui pousse chaque espèce et chaque individu à détruire son prochain, lorsque celui-ci devient obstacle ou concurrent, pour mieux persévérer dans l'être. L'Histoire humaine nous montre que les peuples n'échappent pas à cette loi, bien au contraire. Toutes les civilisations de la Terre n'ont cessé de s'opprimer les unes les autres, de se détruire, de s'asservir, obéissant ainsi, chacune à part, à la Volonté de Puissance qui commande le monde visible.

De ce point de vue, nous dit Nietzsche, la Volonté de Puissance est comme une « mer de forces en tempête ", dont nous avons l'écho en nous-mêmes, et ce à travers la violence de notre vie pulsionnelle (Nietzsche 1886, 37). Elle est innocente, " par-delà le bien et le mal », car elle n'a nulle intention, ni bonne ni mauvaise. Et pour elle, la question " pourquoi y a-t-il quelque chose plutôt que rien? » n'a strictement aucun sens. Elle est comme ces vagues qui, dans l'océan déchaîné, se détruisent elles-mêmes, tout en créant perpétuellement de nouvelles vagues, c'est-à-dire de nouvelles formes, en particulier à travers l'évolution des espèces, lesquelles affirment par leur diversité même la puissance de son expansion. Puissance d'auto-organisation et d'autodestruction, la Volonté de Puissance ne s'inscrit dans aucune finalité. Sous son impulsion, la mort, la cruauté et la guerre trouvent leur place dans l'existence concrète et le «problème du mal », hantise des théologiens, ne jure plus avec le monde créé : il se dissout dans ce que Nietzsche appelé l'innocence du Devenir.

Il faut dire ici combien cette doctrine philosophique épouse les résultats de la science darwinienne, y compris dans ses prolongements les plus contemporains. Nous savons désormais que l'homme s'inscrit dans une longue chaîne évolutive. Avant lui, même, il y a quelques soixante-cinq millions d'années, régnaient des êtres terrifiants, les dinosaures. Si un Dieu omnipotent avait créé l'Être en vue de l'homme, pourquoi auraitil d'abord investi la Terre de ces monstres? Avec la pensée évolutionniste, c'est désormais la nature qui est au premier plan. Quelles sont ses intentions? Quel but poursuit-elle? On a vu que Nietzsche répondait à cette question en la disqualifiant, 
puisque la Volonté de Puissance se meut chez lui sans but. Fidèle à l'enseignement de Darwin, Nietzsche nous a rappelé que nous manquions surtout de "sens historique ", faisant de la figure transitoire de l'homme, un absolu, une essence autour de laquelle nous avions organisé le sens de l'Univers, alors que cette figure n'est que l'instantané d'un flux évolutif appelé à disparaître. C'est là aussi la leçon du Darwinisme, leçon que retiendront tous les existentialismes philosophiques du $\mathrm{XX}^{\mathrm{e}}$ siècle.

\section{BIBLIOGRAPHIE}

Aristote. La Politique. Trad. Tricot. Paris : Vrin, 1962.

---. Physique. Trad. Pellegrin. Paris : Garnier Flammarion, 1999.

Darwin, Charles. Charles Darwin's Notebooks, 1836-1844. Geology, Transmutation of Species, Metaphysical Enquiries. Cambridge: Cambridge University Press, 1987.

---. La Filiation de l'Homme. 1874. Trad. Michel Prum, Patrick Tort. Paris : Syllepse, 1999.

---. On the Origin of Species by Means of Natural Selection, or the Preservation of Favoured Races in the Struggle for Life. London: John Murray, 1859.

Descartes, René. Discours de la Méthode. 1637. Paris : Garnier Flammarion, 2000.

---. Traité de l'homme. 1648. Bibliothèque de la Pléiade. Paris : Gallimard, 1953.

Jacob, François. Le Jeu des possibles, essai sur la diversité du vivant. Paris : Fayard, 1981.

Kant, Emmanuel. Critique de la faculté de juger. 1790. Trad. Philonenko. Paris : Vrin, 1993.

---. Projet de paix perpétuel. 1795. Trad. Marcuzzi. Paris : Vrin, 2007.

Leibniz, Gottfried Wilhelm. Principes de la nature et de la grâce. 1714. Paris : Garnier Flammarion, 1999.

Newton, Isaac. Philosophae naturalis Principia Mathematica. 1687. London: Cambridge University Press, 1972.

Nietzsche, Friedrich. Par-delà bien et mal. 1886. Trad. Colli-Montinari. Paris : Gallimard, 1987.

Rousseau, Jean-Jacques. Emile. 1762. Paris : Flammarion, 1993.

\section{NOTES}

1. Aristote ajoute encore, dans La Politique : "Se suffire à soi-même, est un but auquel tend toute production de la nature et cet état est aussi le plus parfait. Il est donc évident que la Cité est au nombre des choses qui existent naturellement, et que l'homme est naturellement fait pour la société politique » (Aristote 1962, 18).

2. Pour Aristote il y a dans la nature trois ordres de recherche : l'immobile (le Premier Moteur qui doit être immobile sinon il serait mu), le mu incorruptible (le ciel) et le mu corruptible (le monde sublunaire) (Aristote 1999, 63). 
3. Dans ce texte fondateur Leibniz écrit: «Jusqu'ici nous n'avons parlé qu'en simples physiciens: maintenant il faut s'élever à la métaphysique, en nous servant du grand principe, peu employé communément, qui porte que rien ne se fait sans raison suffisante, c'est-à-dire que rien n'arrive sans qu'il soit possible à celui qui connaîtrait assez les choses de rendre une raison qui suffise pour déterminer pourquoi il en est ainsi, et non pas autrement. Ce principe posé, la première question qu'on a droit de faire sera: pourquoi il y a plutôt quelque chose que rien? " (Leibniz 21).

4. C'est en particulier la démarche de Jean-Jacques Rousseau dans Emile, IV, « La profession de foi du vicaire savoyard » (Rousseau 289).

5. Dans un texte demeuré célèbre, qui clôt en 1713 la deuxième édition des Philosophae naturalis Principia Mathematica, et que Newton intitule le scholium generale, le physicien anglais nous avertit de sa conception des choses, qui s'efforce de réintroduire la sagesse de Dieu au sein même de la nature. Pour lui, Dieu est cette présence constante sans laquelle le monde ne saurait persister dans l'être. Il est cet « esprit subtil » qui pénètre à travers tous les corps solides, et qui est caché dans leur substance. Et c'est par la force et l'action de cet esprit, l'esprit de Dieu, que les particules des corps s'attirent mutuellement aux plus petites distances, dans les phénomènes de gravitation (Newton 387-393). Rien n'est plus éloigné de Darwin que cette articulation du mécanisme naturel au finalisme divin.

6. Michel Prum a travaillé en particulier à une nouvelle traduction de The Descent of Man, de Charles Darwin, avec Patrick Tort (Darwin 1999). Il a donné une conférence au Muséum d'Histoire Naturelle de Toulouse, le 24 mars 2009, (« Darwin a-t-il été trahi par ses traducteurs?»), qui reprenait la problématique de la pollution par le langage de la finalité dans la terminologie darwinienne.

\section{RÉSUMÉS}

La théorie darwinienne de l'évolution a remis en question le concept-clef qui avait permis aux doctrines philosophiques les plus importantes de penser la nature : le concept de finalité. Elle nous enseigne que seules demeurent, au sein de la variabilité, les espèces les plus aptes à survivre à l'environnement et à la lutte pour la vie contre d'autres espèces. Les autres sont impitoyablement éliminées, et Darwin nomme ce processus d'élimination « sélection naturelle ». Or cette sélection est un pur mécanisme, ce qui signifie que là ne s'y repèrent aucune finalité, aucune intention, aucune planification, mais bien plutôt de la contingence et du hasard. Darwin est donc celui qui a débarrassé l'idée de nature de celle de finalité. Pourtant, dans l'exposition même de sa théorie, il a dû constamment se battre contre la réintroduction de ce concept dans sa manière de parler de la nature. Tout se passe comme si la question de la finalité avait fini par resurgir, venant polluer le discours même qui avait tenté d'en éliminer la pertinence. Comment interpréter cette persistance?

The Darwinian theory of evolution questions the major concept which had allowed the most important philosophical doctrines to conceptualize nature: finality. It teaches us that the only species to hold their ground within variability are the fittest to survive in the environment, 
winning the "struggle for life" against other species. The others are pitilessly eliminated, and Darwin names this process of elimination natural selection. However, this selection is a pure mechanism, which means that there is no finality in it, no intention, no planning, but rather contingency and chance. Darwin is thus the one who separated the ideas of nature and finality. Still, the presentation of his theory shows Darwin's constant fight against the reintroduction of this concept in his dealing with nature. It is as though the issue of finality had finally reasserted itself, coming to pollute the discourse which had tried to displace it. How can we interpret this persistence?

INDEX

Keywords : Darwin, Darwinism, finality, philosophy

Mots-clés : darwinisme, évolution, philosophie, Darwin

\section{AUTEURS}

\section{PHILIPPE SOLAL}

PRAG

Institut National des Sciences Appliquées (INSA)

psolal@neuf.fr 